\title{
Thermal Effusivity Calibration Procedure for Thermal Microscope
}

\author{
Shugo Miyake, ${ }^{1 *}$ Tetsuya Ohtsuki, ${ }^{2}$ Takaaki Awano, ${ }^{2}$ \\ Kimihito Hatori, ${ }^{2}$ and Makoto Sekine ${ }^{2}$ \\ ${ }^{1}$ Department of Mechanical Engineering, Kobe City College of Technology \\ 8-3 Gakuenhigashi-machi, Kobe 651-2194, Japan \\ ${ }^{2}$ Hudson Laboratory, Bethel Co., Ltd., 9-3 Yamatocho, Tsuchiura, Ibaraki 300-0036, Japan
}

(Received August 25, 2018; accepted November 5, 2018)

Keywords: thermoreflectance, thermal microscope, thermal effusivity, molybdenum thin film

In this paper, a thermal effusivity calibration procedure is proposed using a numerical calculation and measurement of a phase lag of a thermoreflectance signal based on a periodic modulation thermoreflectance method for a thermal microscope. To improve the accuracy of the estimated thermal effusivity, the profile determination of the heating and probing laser beams and the numerical calculation of phase lags with a two-layer model in a threedimensional heat conduction equation are carried out. Results of the experiments of beam profile determination and numerical calculation showed that the estimated values of thermal effusivities are in good agreement with the standard values of silicon, germanium, and Pyrex ${ }^{\circledR}$ samples. We are convinced that the use of a thermal microscope with numerical calibration is becoming an accurate and precise thermal property measurement technique for the microscale range.

\section{Introduction}

The characterization of the thermal property and heat transport of solid materials for the microscale range is becoming an important subject for improving thermal design in diverse fields, such as materials, electronics, physics, and mechanics, and because of that, an exothermic problem is becoming serious with the increasing number of multifunctionalized and highly integrated devices. Accordingly, the requirement of a thermal property measurement technique for the microscale range is increasing to solve this exothermic problem. To meet the demands of the characterization of the thermal property with microscale spatial resolution, several techniques have been proposed. In general, a transient method of using a focused laser beam has been employed to generate an instantaneous heat source on the desired sample surface. The most popular technique in the field of thermophysics is the thermoreflectance method. This method was proposed for the investigation of the thermal property of a metal material by Rosencwaig et al. in 1985. ${ }^{(1)}$ The thermoreflectance method is based on the measurement of the temperature dependence of the reflectivity of a sample surface. The sample should be deposited on a metal thin film as a requisite reflector for the following reasons. To generate the

*Corresponding author: e-mail: miyake@kobe-kosen.ac.jp

https://doi.org/10.18494/SAM.2019.2120 
instantaneous heat on the sample surface, an ultrafast pulse laser beam or a periodic modulated laser beam is commonly used. Over the past few decades, a considerable number of studies have been conducted on the thermoreflectance method. ${ }^{(2-21)}$ In all of those studies, the reflector is the essential part. This is because the reflector plays an important role as not only a reflector but also an absorber to propagate the heat flux into the target sample. An example of a typical reflector material is a molybdenum thin film deposited by physical vapor deposition. The behavior of the thermoreflectance signal in periodic modulation heating has been studied by numerical calculation and experiments. ${ }^{(22-24)}$ These studies revealed the characteristics of the thermoreflectance signal as a temperature response at the center of the heating spot as a three-dimensional heat conduction equation in the cylindrical coordinate system. However, without the correct values of the thermal and physical properties of the reflector material under initial conditions, that is, the thermal conductivity $(\lambda)$, specific heat $(C)$, and density $(\rho)$, it is impossible to solve the problem precisely. Moreover, strictly speaking, we must consider the thermal resistance between the reflector thin film and the target sample along with the thermal conductivity of the reflector in the numerical calculation. Nevertheless, the measurement of the thermal conductivity of the reflector material, which involves thermal diffusivity, specific heat, and density, is not easy owing to the need for time-domain thermoreflectance equipment with an ultrafast pulse laser beam module. ${ }^{(2,3,5)}$ In addition, there is the problem that the thermal resistance between the reflector and the target sample must be estimated at each preparation step of the sample. Namely, by understanding the apparent thermal conductivity, including the thermal conductivity of the reflector and the thermal resistance between the reflector and each target sample, through numerical calculation and thermoreflectance measurement, we can solve the thermal property of the target sample accurately.

For the reasons mentioned above, we attempt to estimate the apparent thermal conductivity of a reflector material, including thermal resistance, by numerical calculation and thermoreflectance measurement using reference silicon ( $\mathrm{Si}$ ), germanium (Ge), and borosilicate glass $\left(\right.$ Pyrex $\left.^{\circledR}\right)$ samples. In this paper, we present an estimation method of the apparent thermal conductivity of a reflector and a procedure for accurate thermal effusivity measurement.

\section{Experiments}

We proposed an estimation procedure of the apparent thermal conductivity of a reflector with the following steps. First, the thermoreflectance method for numerical calculation is modeled. Secondly, the effective radii of the heating and probing laser beams of the thermoreflectance equipment (Thermal microscope, Bethel) are determined by the knife-edge method. Then, the numerical calculation is carried out with various temporary thermal conductivities of the reflector thin film (molybdenum, Mo). Finally, by comparing the analyzed and measured phase lags obtained by numerical calculation and phase measurement using a thermal microscope, respectively, we can determine the apparent thermal conductivity of the deposited Mo thin film on the target sample. 


\subsection{Calculation model for thermoreflectance method}

Figure 1 shows the schematic model of the thermoreflectance method for numerical calculation, which is based on previous studies. ${ }^{(23,24)}$ This model consists of two layers of solids and air. The subscripts 0,1 , and 2 denote the air, the Mo layer, and the sample layer, respectively. $a$ and $\sigma$ are the radii of the heating and probing laser beams, which have the Gaussian spatial distribution, on the Mo film surface, respectively. Incidentally, the radius of the probing laser beam has a major effect on the spatial resolution of the thermal microscope. The radii are determined by the distance between the points where the laser power is $e^{-2}$. Note that it is important to align the two coaxial laser beams perfectly.

\subsection{Calculation principle}

In the thermoreflectance method, we are interested in the temperature response at the center of the heating point. The temperature response is determined as a phase lag, which is a difference between the phases of the heating laser beam and the determined thermoreflectance signal using the thermal microscope. The principle of the calculation method considering the relationship between the volumetric heat capacity and the thermal effusivity is described in related previous papers. ${ }^{(23,24)}$ Thus, only the key points of the calculation method are described below.

This calculation model is constructed using the three-dimensional heat conduction equation in the cylindrical coordinate system. The intensity of the heating laser beam is sinusoidally modulated with the frequency $f=\omega / 2 \pi$. A heat flux $q(r, t, z)$ is generated by the heating laser beam on the surface of the Mo layer, and it is represented as

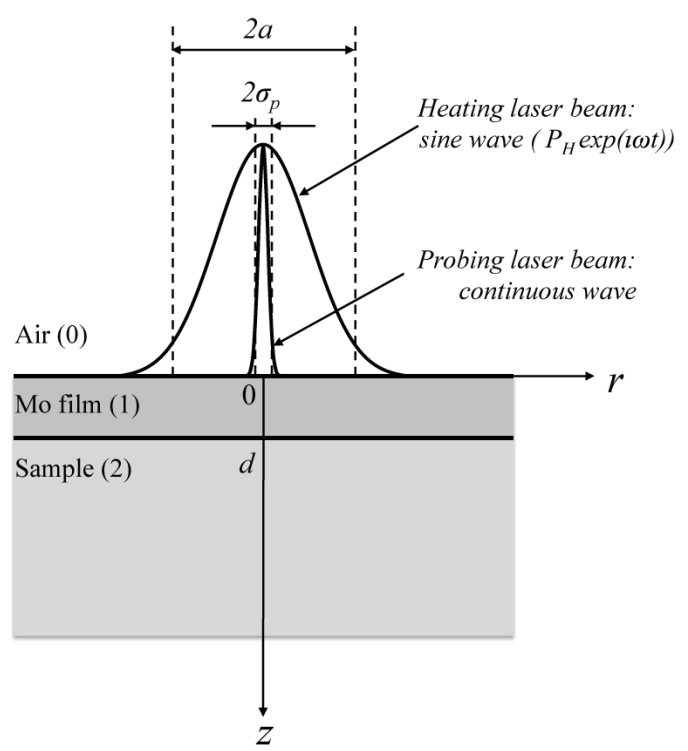

Fig. 1. Schematic model of thermoreflectance method. 


$$
q(r, t, z)=\frac{2 P \alpha}{\pi a^{2}} \exp (-\alpha z) \exp \left(-\frac{2 r^{2}}{a^{2}}\right) \exp (i \omega t)+c . c .
$$

where $t$ is time, $P$ is the output power, $\alpha$ is the absorption coefficient of the Mo film for the wavelength of the heating laser, and c.c. is the complex conjugate. The temperature response of the Mo film surface, $T_{1}(r, t)$, is represented by

$$
\begin{gathered}
T_{1}(r, t)=\left[\int_{0}^{\infty} \tau(p, z) p J_{0}(p r) d p\right] \exp (i \omega t)+c . c ., \\
\tau(p, z)=\Gamma(p) e^{-\alpha z}+A(p) e^{\beta_{1} z}+B(p) e^{-\beta_{1} z}, \\
\Gamma(p)=\frac{P \alpha}{2 \pi \lambda_{1}} \cdot \frac{\exp \left[-(p a)^{2} / 8\right]}{\beta_{1}^{2}-\alpha^{2}}, \\
\beta_{i}^{2}=p^{2}+i \omega / k_{i}(i=0,1,2), \\
k_{2}=b^{2} /\left(\rho C_{p}\right)^{2},
\end{gathered}
$$

where $J_{0}$ is the Bessel function of the first kind of order 0 , and $k$ and $\lambda$ are the thermal diffusivity and thermal conductivity, respectively. A subscript $i=0,1,2$ denotes the layer of air, the Mo film, and the sample, as shown in Fig. 1, respectively. In Eq. (3d), $b, \rho$, and $C_{p}$ are the thermal effusivity, density, and specific heat of the sample, respectively. Incidentally, $\rho C_{p}$ is the volumetric heat capacity. In Eq. (3a), $A(p)$ and $B(p)$ denote the amplitudes of the thermal waves propagating in the positive and negative $z$ directions, respectively. These are determined by the following boundary conditions:

$$
\begin{gathered}
\left.\lambda_{0} \frac{\partial T_{0}}{\partial z}\right|_{z=0}=\left.\lambda_{1} \frac{\partial T_{1}}{\partial z}\right|_{z=0},\left.\quad \lambda_{1} \frac{\partial T_{1}}{\partial z}\right|_{z=d}=\left.\lambda_{2} \frac{\partial T_{2}}{\partial z}\right|_{z=d} \\
\left.T_{0}\right|_{z=0}=\left.T_{1}\right|_{z=0},\left.T_{1}\right|_{z=d}=\left.T_{2}\right|_{z=d} .
\end{gathered}
$$

Then, $A(p)$ and $B(p)$ are represented as

$$
A(p)=\left[(1-g)(\gamma-c) e^{-\alpha l}-(\gamma+g)(1+c) e^{\beta_{1} l}\right] \cdot \Gamma(p) / H(p),
$$




$$
B(p)=\left[(1+g)(\gamma-c) e^{-\alpha l}-(\gamma+g)(1-c) e^{\beta_{1} l}\right] \cdot \Gamma(p) / H(p),
$$

where

$$
\begin{gathered}
H(p)=(1+g)(1-c) e^{\beta_{1} l}-(1-g)(1-c) e^{\beta_{1} l} \\
g=\lambda_{0} \beta_{0} / \lambda_{1} \beta_{1}, c=\lambda_{2} \beta_{2} / \lambda_{1} \beta_{1}, \gamma=\alpha / \beta_{1} .
\end{gathered}
$$

The surface temperature can be calculated by integrating Eq. (2) with Eqs. (3a)-(5d). The thermoreflectance signal $R(t)$ is obtained from the surface temperature $T(r, t)$ and the probing laser beam profile $f_{p}(r)$ using a convolution integral:

$$
\begin{gathered}
f_{p}(r)=\frac{P_{p}}{\pi \sigma_{p}} \cdot e^{\left[-\frac{r^{2}}{\sigma_{p}^{2}}\right],} \\
R(t)=c_{n o r m} 2 \pi \int_{0}^{\infty} T_{1}(r, t) \cdot f_{p}(r) r d r,
\end{gathered}
$$

where $c_{\text {norm }}$ is the normalization constant and $P_{p}$ and $\sigma_{p}$ represent the power and $e^{-2}$ radius of the probing laser beam, respectively. Then, the phase lag $\theta$ is represented as

$$
\theta=\operatorname{Arg}[R(0)]
$$

Accordingly, by substituting the designated thermal properties of the Mo film and the sample into these equations, the resulting matrix chart of the thermal effusivity and the volumetric heat capacity as functions of the phase lag enable us to determine the thermal effusivity of an unknown sample through an interpolation of the measured phase lag.

\subsection{Laser beam profiling}

Laser beam profiling is performed by the knife-edge method. Figure 2 shows the schematic of the laser beam profiling method. A silicon photodiode is mounted on the electric $x$-stage, the displacement resolution of which is $100 \mathrm{~nm}$. The effective photosensitive area of the silicon photodiode is $4 \times 4 \mathrm{~mm}^{2}$. A molybdenum film over $500 \mathrm{~nm}$ thick is deposited on half the effective photosensitive area as a shielding film by the DC-sputtering method. A thin silicon wafer chip protected the nondeposited area during deposition, and the boundary between the deposited and nondeposited area is the so-called knife edge. The thickness of the Mo 


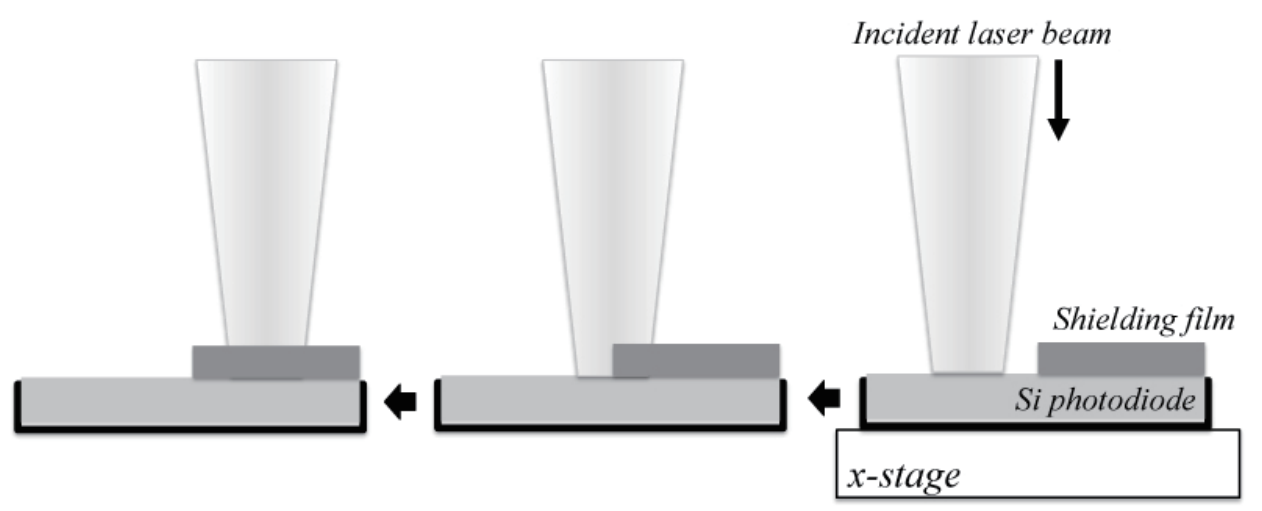

Fig. 2. Schematic of laser beam profiling method.

shield film is sufficient to absorb almost $100 \%$ of the irradiated laser beam. When the total intensity of the irradiated laser beam is received by the shielding film, the output voltage of the photodiode is still the background level. While moving the $x$-stage, the laser beam steadily irradiates the exposed effective photosensitive area. The heating and probing laser beams are profiled using the knife-edge apparatus with the $x$-stage moving $25 \mu \mathrm{m}$ in $100 \mathrm{~nm}$ steps (in the range of $\pm 12.5 \mu \mathrm{m}$ from the deposited boundary). These measured profiles are differentiated and fitted by the Gaussian distribution function. Figure 3 shows the results of the measured and Gaussian-fitted profiles of the heating laser beam. The unit along the vertical axis is arbitrary, and that along the horizontal axis is the relative knife-edge position $x$. The radius of the laser beam is determined using the full width at $e^{-2}$ during the analysis of the Gaussian fitting profile. The resulting radius of the heating laser beam is $5.05 \mu \mathrm{m}$ and that of the probing laser beam is $1.00 \mu \mathrm{m}$; the spatial resolution is estimated to be $2.00 \mu \mathrm{m}$.

\section{Numerical Calculation}

On the basis of the above-mentioned theory and required thermal and physical parameters, a numerical calculation was carried out. Table 1 shows the calculation conditions in this study. Here, as an initial condition, the thermal conductivities of the Mo film, i.e., 5, 10, and $50 \mathrm{Wm}^{-1} \mathrm{~K}^{-1}$, are used. These values are taken from previous studies. ${ }^{(23,24)}$ Note that the thermal conductivity of the Mo film can be controlled by adjusting the sputtering conditions such as the kind of gas for plasma generation, gas pressure, back pressure, and substrate temperature. In particular, the thermal diffusivity is strongly affected by these parameters. ${ }^{(25)}$ In this calculation, the range of thermal effusivity is from 0 to $20000 \mathrm{Js}^{-0.5} \mathrm{~m}^{-2} \mathrm{~K}^{-1}$ and also, the volumetric heat capacity is between 1.0 and $4.0 \times 10^{6} \mathrm{Jm}^{-3} \mathrm{~K}^{-1}$, which conform to the range of thermal properties of practical materials.

\section{Results and Discussion}

Figure 4 shows the results of the numerical calculation, i.e., phase contours of the thermal properties as a function of the thermal conductivity of the Mo film. The vertical axis is thermal 


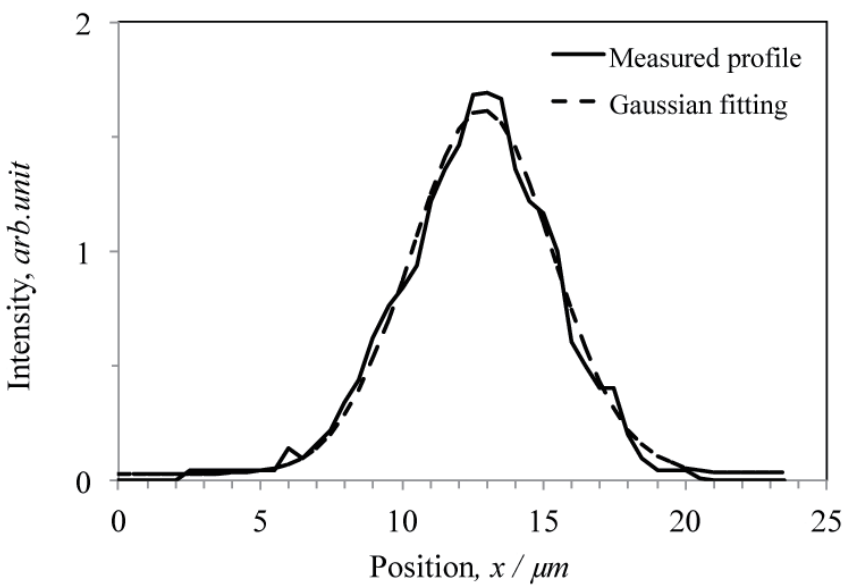

Fig. 3. Results of the measured and Gaussian-fitted profiles of the heating laser beam.

Table 1

Thermal properties and optical conditions for calculation.

\begin{tabular}{lcc}
\hline Material & Parameter & Value \\
\hline Mo & Thickness, $d$ & $108.9 \mathrm{~nm}^{-1} \mathrm{~K}^{-1}$ \\
& Thermal conductivity, $\lambda$ & $5,10,50 \mathrm{Wm}^{-1}-\mathrm{K}^{-1}$ \\
& Specific heat, $C_{M o}$ & $248 \mathrm{Jkg}^{-1} \mathrm{~K}$ \\
& Density, $\rho$ & $7640 \mathrm{kgm}^{-3}$ \\
Air & Absorption coefficient, $\alpha$ & $30 \mathrm{~m}^{-1}$ \\
& Thermal conductivity, $\lambda$ & $0.0259 \mathrm{Wm}^{-1} \mathrm{~K}^{-1}$ \\
& Specific heat, $C_{M o}$ & $0.001 \mathrm{Jkg}^{-1} \mathrm{~K}^{-1}$ \\
Heating laser & Density, $\rho$ & $1.184 \mathrm{kgm}^{-3}$ \\
& Frequency, $f$ & $1 \mathrm{MHz}$ \\
& Power, $P$ & $30 \mathrm{~mW}$ \\
Probing laser & Radius, $a$ & $5.045 \mu \mathrm{m}$ \\
\hline
\end{tabular}

$5 \mathrm{Wm}^{-1} \mathrm{~K}^{-1}$

$10 \mathrm{Wm}^{-1} \mathrm{~K}^{-1}$

$50 \mathrm{Wm}^{-1} \mathrm{~K}^{-1}$

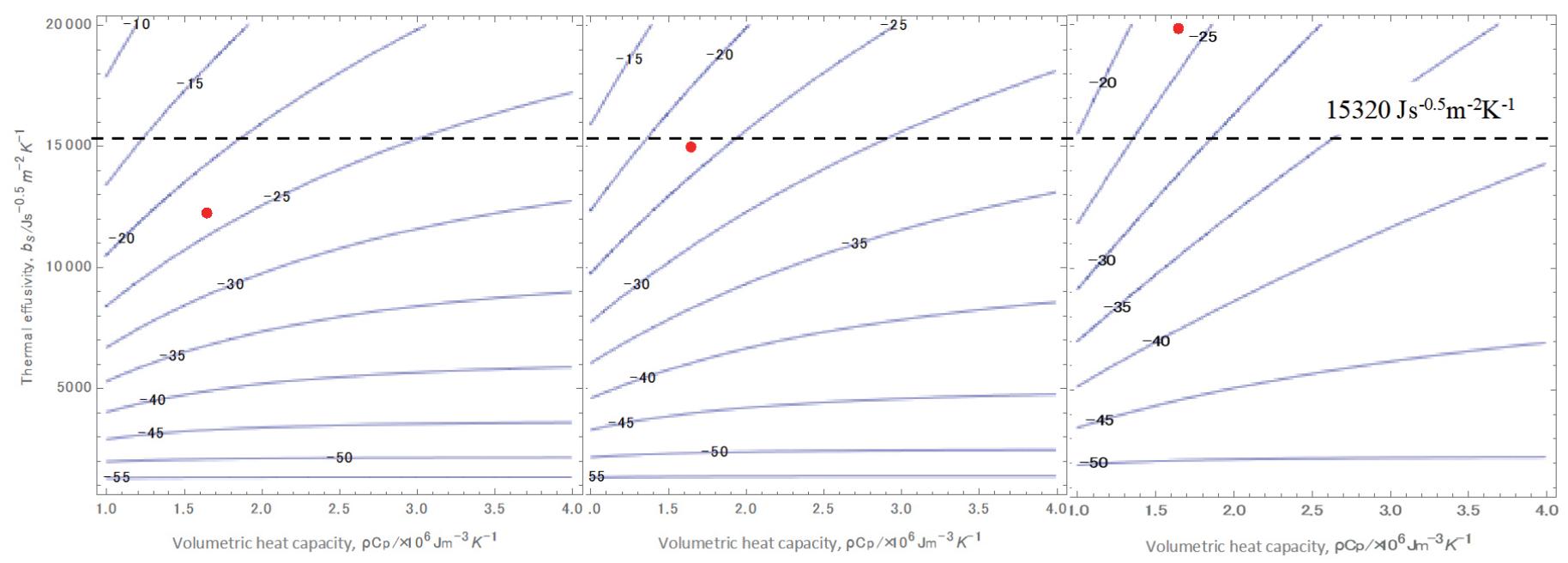

Fig. 4. (Color online) Contour plots of phase lag matrix as a function of thermal conductivity of Mo film on silicon. 
effusivity, and the horizontal axis is volumetric heat capacity. Herein, the solid circle denotes the phase measurement result of the silicon sample; the value of the phase lag is $-23.18^{\circ}$, as determined using the thermal microscope, where the measurement and optical conditions take into account the conditions of the numerical calculation. The plotted positions of the thermal effusivity and volumetric heat capacity of the used silicon sample were taken from previous studies. $^{(23,24)}$ A dashed line with superimposed contours shows the value of the thermal effusivity of the reference silicon. Incidentally, the apparent thermal conductivity of the Mo film deposited on the silicon sample by DC sputtering is naturally unknown at that moment. Thus, we show the matrix of the phase contours with various thermal conductivities of the Mo film in Fig. 4. The result of calculation using the value of $5 \mathrm{Wm}^{-1} \mathrm{~K}^{-1}$ of the Mo film shows that the estimated thermal effusivity of the silicon is $12268 \mathrm{Js}^{-0.5} \mathrm{~m}^{-2} \mathrm{~K}^{-1}$, which is lower than the expected value of $15321 \mathrm{Js}^{-0.5} \mathrm{~m}^{-2} \mathrm{~K}^{-1}$. The result of using $10 \mathrm{Wm}^{-1} \mathrm{~K}^{-1}$ is $14989 \mathrm{Js}^{-0.5} \mathrm{~m}^{-2} \mathrm{~K}^{-1}$, which is also lower than the expected value. In contrast, the result of using $50 \mathrm{Wm}^{-1} \mathrm{~K}^{-1}$ of the Mo film is $19856 \mathrm{Js}^{-0.5} \mathrm{~m}^{-2} \mathrm{~K}^{-1}$, which is higher than the expected value. This is because the thermal conductivity of the Mo film is unsuitable for the numerical calculation. By optimizing the apparent thermal conductivity of the Mo film by a partial parameter change measurement, we finally determined the thermal conductivity of the deposited Mo film for this measurement to be $11 \mathrm{Wm}^{-1} \mathrm{~K}^{-1}$, which is in good agreement with the expected value, as shown in Fig. 5. Hence, it is found that the apparent thermal conductivity of the Mo film can be estimated by numerical calculation and standard sample measurement. Furthermore, phase lags of the Pyrex ${ }^{\circledR}$ and germanium samples deposited on the Mo film at the same time as the silicon preparation are measured using the thermal microscope and analyzed with the calculated matrix results. These thermal properties are shown in Table 2. Figure 6 shows plots of these results; there is good agreement between the measured phase lags and the reference values. The estimated apparent thermal conductivity of the Mo film is not a universal value.

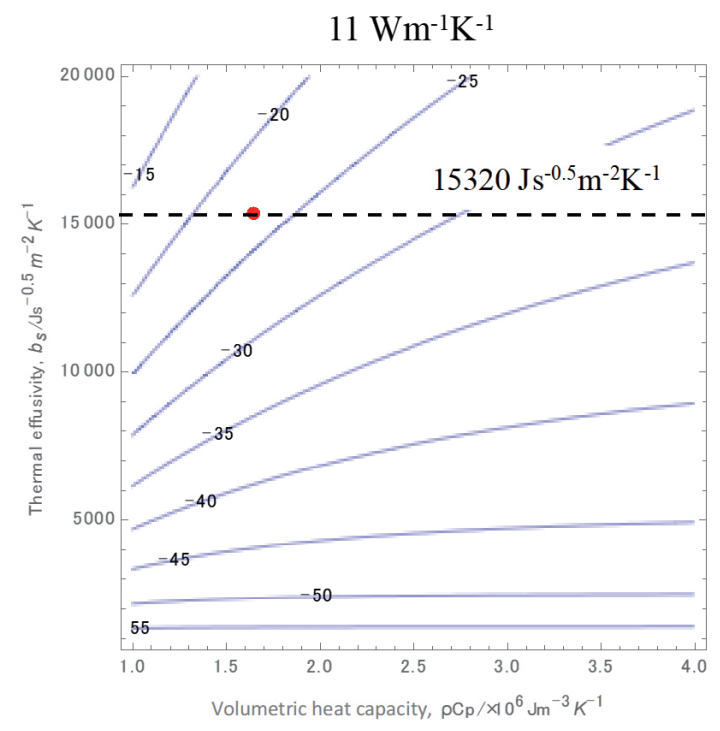

Fig. 5. (Color online) Optimized contour plots of phase lag matrix of Mo film on silicon. 
Table 2

Thermal properties of samples. ${ }^{(23)}$

\begin{tabular}{lccc}
\hline Material & $\begin{array}{c}\text { Specific heat, } C_{p} \\
\left(\mathrm{Jkg}^{-1} \mathrm{~K}^{-1}\right)\end{array}$ & $\begin{array}{c}\text { Density, } \rho \\
\left(\mathrm{kgm}^{3}\right)\end{array}$ & $\begin{array}{c}\text { Thermal effusivity, } b \\
\left(\mathrm{Js}^{-0.5} \mathrm{~m}^{-2} \mathrm{~K}^{-1}\right)\end{array}$ \\
\hline Silicon & 709 & 2322 & 15321 \\
Germanium & 324 & 5213 & 10428 \\
Pyrex $^{\circledR}$ & 773 & 2218 & 1417 \\
\hline
\end{tabular}

Pyrex

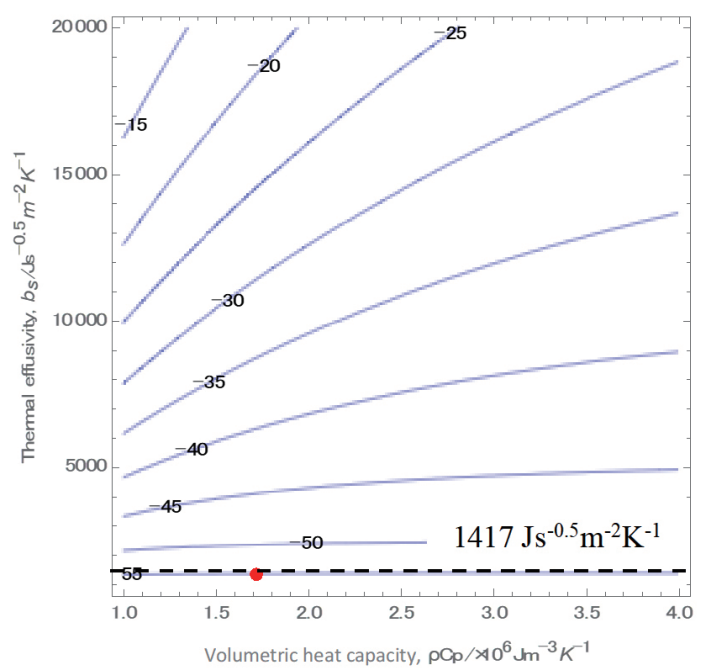

Ge

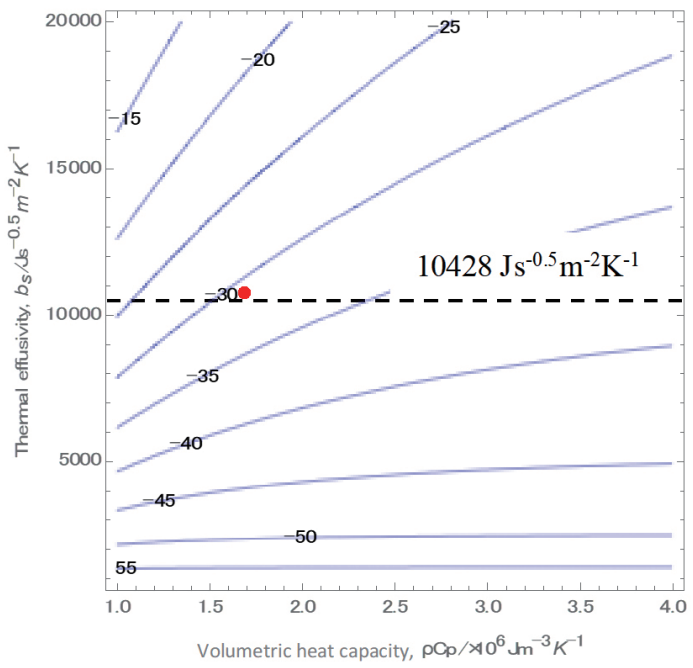

Fig. 6. (Color online) Contour plots of Pyrex ${ }^{\circledR}$ and Ge sample with the thermal conductivity of the Mo film of 11 $\mathrm{Wm}^{-1} \mathrm{~K}^{-1}$.

This is because, as shown previously, the apparent thermal conductivity involves the thermal conductivity of the Mo film and the thermal resistance between the Mo film and the target sample. Therefore, we have to verify the relationship between the thermal conductivity of the Mo film and the DC-sputtering conditions by a number of experiments. However, it is very difficult to estimate precisely only the thermal conductivity (excluding the thermal resistance) of the Mo film at every measurement by the time-domain thermoreflectance method because a special sample, which is not needed for the thermal microscope owing to the different measurement and analysis principle, needs to be prepared. On the other hand, an advantage of the proposed calibration method is that it enables us to use the same sample to estimate the apparent thermal conductivity of the reflector Mo; the measurement and analysis methods are easier than other microscale thermal measurement methods. From what has been revealed through the calculation and measurement, we can conclude that the validity of the thermoreflectance calibration method, including the estimation of the thermal conductivity of the Mo film, has been shown in this study. In Fig. 7, we summarize the procedure for thermal effusivity analysis using the thermal microscope based on the numerical calculation and phase lag measurement. 


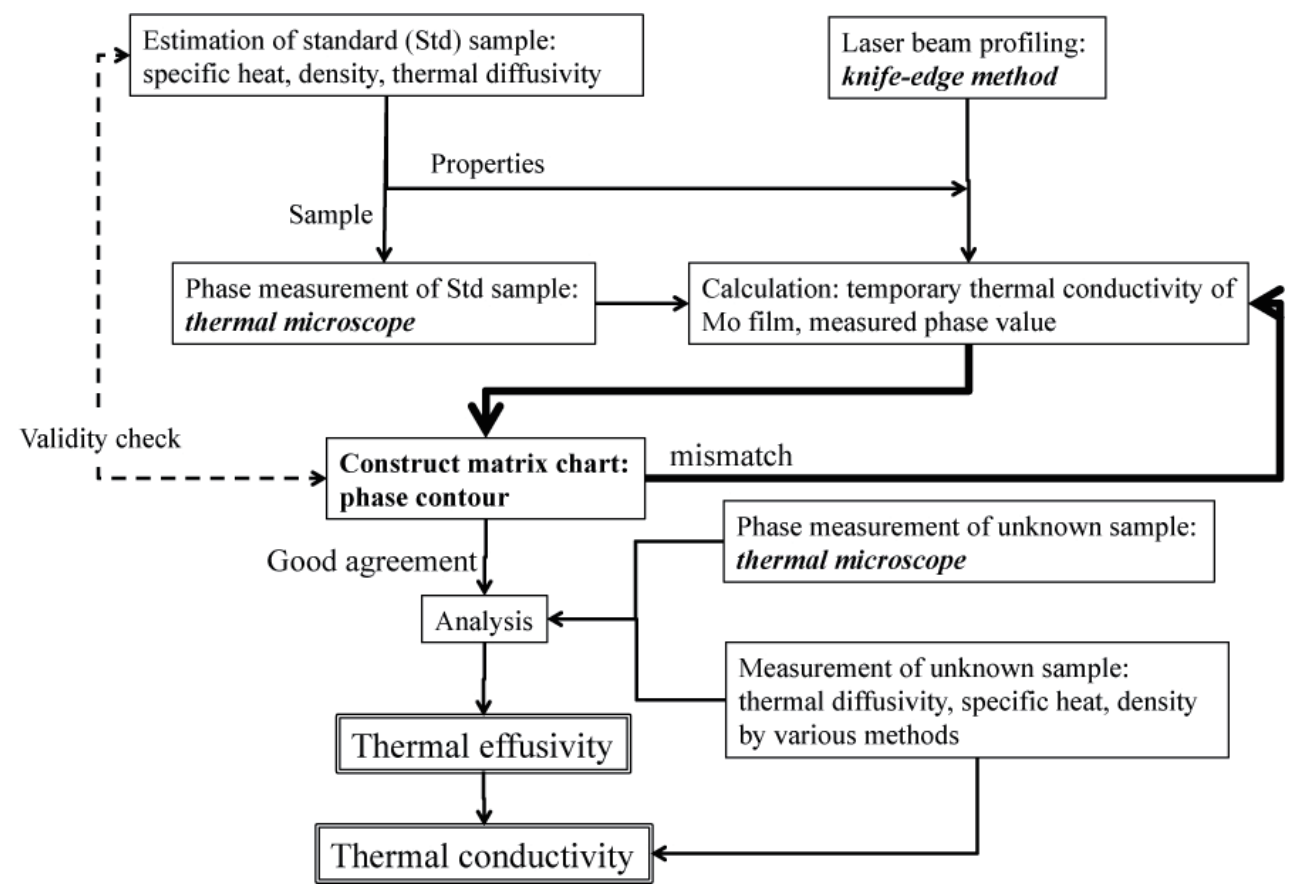

Fig. 7. Thermoreflectance calibration procedure for thermal microscope.

\section{Conclusions}

In this paper, we proposed a thermal effusivity analysis method using a thermal microscope with several steps using a laser beam profiler and numerical calculation. This method enables us to estimate the apparent thermal conductivity to correct the phase matrix chart for a sample with unknown thermal properties. The thermal microscope based on the periodic modulation heating thermoreflectance method is becoming a powerful tool delving into a more precise and narrow scale region in future work.

\section{Acknowledgments}

This research was supported by a grant-in-aid of the Hirosawa Foundation for Technology (H29).

\section{References}

1 A. Rosencwaig, J. Opsal, W. L. Smith, and D. L. Willenborg: Appl. Phys. Lett. 46 (1985) 1013.

2 N. Taketoshi, T. Baba, and A. Ono: Jpn. J. Appl. Phys. 38 (1999) L1268H.

3 N. Taketoshi, T. Baba, and A. Ono: Meas. Sci. Technol. 12 (2001) 2064.

4 B. C. Daly, H. J. Maris, A. V. Nurmikko, M. Kuball, and J. Han: J. Appl. Phys. 92 (2002) 3820.

5 N. Taketoshi, T. Baba, E. Schaub, and A. Ono: Rev. Sci. Instrum. 74 (2003) 128.

6 K. Hatori, N. Taketoshi, T. Baba, and H. Ohta: Rev. Sci. Instrum. 76 (2005) 114901.

7 R. Kato and I. Hatta: Int. J. Thermophys. 26 (2005) 179.

8 M. Kuwahara, O. Suzuki, N. Taketoshi, Y. Yamakawa, T. Yagi, P. Fons, K. Tsutsumi, K. Suzuki, T. Fukaya, and J. Tominaga: J. Appl. Phys. 45 (2006) 1419. 
9 H. Fukuyama, T. Yoshimura, H. Yasuda, and H. Ohta: Int. J. Thermophys. 27 (2006) 1760.

10 C. Fretigny, J. P. Roger, V. Reita, and D. Fournier: J. Appl. Phys. 102 (2007) 116104.

11 R. Kato and I. Hatta: Int. J. Thermophys. 29 (2008) 2062.

12 S.-K. Lee, I. Yamada, S. Kume, H. Nakano, K. Hatori, G. Matsui, and K. Watari: J. Ceram. Soc. 116 (2008) 1260.

13 Y. Okamoto, J. Saeki, T. Ohtsuki, and H. Takiguchi: Appl. Phys. Express 1 (2008) 117001.

14 S. Miyake, T. Kita, A. Miyake, K. Ikeda, and H. Takamatsu: Rev. Sci. Instrum. 80 (2009) 124901.

15 K. Hatori, K. Suzuki, H. Fukuyama, and H. Ohta: Heat Transfer-Asian Res. 38 (2009) 57.

16 R. Kato, Y. Xu, and M. Goto: Jpn. J. Appl. Phys. 50 (2011) 046602.

17 R. Kato, Y. Xu, and M. Goto: Jpn. J. Appl. Phys. 50 (2011) 106602.

18 H. Shibata, H. Ohta, T. Nemoto, S. Nagasaka, Y. Waseda, K. Fujii, and K. T. Jacob: High Temp. Process. 29 (2011) 515.

19 S. Miyake, S. Kanetsuki, K. Morino, J. Kuroishi, and T. Namazu: Jpn. J. Appl. Phys. 54 (2015) 06FP15.

20 S. Miyake, T. Kato, H. Taguchi, and T. Namazu: Jpn. J. Appl. Phys. 55 (2016) 06 GP08.

21 S. Miyake, K. Hatori, T. Ohtsuki, T. Awano, and M. Sekine: Jpn. J. Appl. Phys. 57 (2018) 06HJ03.

22 G. Matsui and H. Kato: Rev. Sci. Instrum. 82 (2011) 034905.

23 H. Ohta, K. Hatori, G. Matsui, T. Yagi, S. Miyake, T. Okamura, R. Endo, R. Okada, K. Morishita, S. Yokoyama, K. Taguchi, and H. Kato: Meas. Sci. Technol. 27 (2016) 115002.

24 S. Miyake, G. Matsui, H. Ohta, K. Hatori, K. Taguchi, and S. Yamamoto: Meas. Sci. Technol. 28 (2017) 075006 .

25 N. Taketoshi, T. Yagi, and T. Baba: Jpn. J. Appl. Phys. 48 (2009) 05EC01. 